\title{
Glucose patterns during an oral glucose tolerance test and associations with future diabetes, cardiovascular disease and all-cause mortality rate
}

\author{
Adam Hulman ${ }^{1,2,3}$ • Dorte Vistisen ${ }^{4}$. Charlotte Glümer ${ }^{5}$ - Michael Bergman ${ }^{6}$. \\ Daniel R. Witte ${ }^{1,2} \cdot$ Kristine Færch $^{4}$
}

Received: 24 May 2017 / Accepted: 7 September 2017 /Published online: 6 October 2017

(C) Springer-Verlag GmbH Germany 2017

\begin{abstract}
Aims/hypothesis In addition to blood glucose concentrations measured in the fasting state and $2 \mathrm{~h}$ after an OGTT, intermediate measures during an OGTT may provide additional information regarding a person's risk of future diabetes and cardiovascular disease (CVD). First, we aimed to characterise heterogeneity of glycaemic patterns based on three time points during an OGTT. Second, we compared the incidences of diabetes and CVD and all-cause mortality rates among those with different patterns.

Methods Our cohort study included 5861 participants without diabetes at baseline from the Danish Inter99 study. At baseline, all participants underwent an OGTT with measurements of plasma glucose levels at 0,30 and $120 \mathrm{~min}$. Latent class mixed-effects models were fitted to identify distinct patterns of glycaemic response during the OGTT. Information
\end{abstract}

Electronic supplementary material The online version of this article (https://doi.org/10.1007/s00125-017-4468-z) contains peer-reviewed but unedited supplementary material, which is available to authorised users.

Adam Hulman

adam.hulman@ph.au.dk

1 Department of Public Health, Aarhus University, Bartholins Allé 2, Building 1260, DK-8000, Aarhus C, Denmark

2 Danish Diabetes Academy, Odense, Denmark

3 Department of Medical Physics and Informatics, University of Szeged, Szeged, Hungary

4 Steno Diabetes Center Copenhagen, Gentofte, Denmark

5 Research Centre for Prevention and Health, Glostrup Hospital, Glostrup, Denmark

6 Division of Endocrinology, Diabetes and Metabolism, NYU School of Medicine, NYU Langone Diabetes Prevention Program, New York, NY, USA regarding incident diabetes, CVD and all-cause mortality rates during a median follow-up time of 11,12 and 13 years, respectively, was extracted from national registers. Cox proportional hazard models with adjustment for several cardiometabolic risk factors were used to compare the risk of diabetes, CVD and all-cause mortality among individuals in the different latent classes.

Results Four distinct glucose patterns during the OGTT were identified. One pattern was characterised by high $30 \mathrm{~min}$ but low $2 \mathrm{~h}$ glucose values. Participants with this pattern had an increased risk of developing diabetes compared with participants with lower $30 \mathrm{~min}$ and $2 \mathrm{~h}$ glucose levels (HR 4.1 [95\% CI $2.2,7.6]$ ) and participants with higher $2 \mathrm{~h}$ but lower $30 \mathrm{~min}$ glucose levels (HR 1.5 [95\% CI 1.0, 2.2]). Furthermore, the all-cause mortality rate differed between the groups with significantly higher rates in the two groups with elevated $30 \mathrm{~min}$ glucose. Only small non-significant differences in risk of future CVD were observed across latent classes after confounder adjustment.

Conclusions/interpretation Elevated 30 min glucose is associated with increased risk of diabetes and all-cause mortality rate independent of fasting and $2 \mathrm{~h}$ glucose levels. Therefore, subgroups at high risk may not be revealed when considering only fasting and $2 \mathrm{~h}$ glucose levels during an OGTT.

Keywords 30 minute post-OGTT glucose $\cdot$ Cardiovascular disease $\cdot$ Latent class modelling $\cdot$ Mortality $\cdot$ Oral glucose tolerance test $\cdot$ Plasma glucose curve $\cdot$ Type 2 diabetes
Abbreviations
$1 \mathrm{hPG} \quad 1 \mathrm{~h}$ plasma glucose
$2 \mathrm{hPG} \quad 2 \mathrm{~h}$ plasma glucose
30minPG 30 min plasma glucose
CVD Cardiovascular disease 
FPG Fasting plasma glucose

ISI $_{0-120} \quad$ Insulin sensitivity index

PG-AUC Area under the plasma glucose curve

PG-iAUC Incremental area under the

plasma glucose curve

\section{Introduction}

Blood glucose concentrations measured in the fasting state and $2 \mathrm{~h}$ after an OGTT are used for the diagnosis of impaired glucose tolerance and type 2 diabetes [1]. Intermediate measures between fasting plasma glucose (FPG) and $2 \mathrm{~h}$ post-load plasma glucose $(2 \mathrm{hPG})$, e.g. $30 \mathrm{~min}$ plasma glucose (30minPG) or $1 \mathrm{~h}$ plasma glucose (1hPG) concentrations, have not received much attention in clinical practice, but epidemiological studies have shown that these may be more predictive of risk for future diabetes than FPG, $2 \mathrm{hPG}$ or $\mathrm{HbA}_{1 \mathrm{c}}$ [2-5]. Furthermore, different features of OGTTderived glucose curves have been associated with diabetes incidence, e.g. time to peak, time to nadir and whether the curve was monophasic or biphasic [3, 6, 7]. Cardiovascular disease (CVD) and all-cause mortality rates have received even less attention in previous studies [8,9]. All previous analyses studying long-term outcomes used predefined characteristics to categorise glucose patterns and they did not take measurement error into account. However, using a data-driven statistical approach, latent class trajectory analysis, we recently showed that individuals with a high glucose peak approximately 45 min after intake of $75 \mathrm{~g}$ glucose, but normal $2 \mathrm{~h}$ glucose levels, had an increased estimated 10 year risk of CVD compared with those with higher $2 \mathrm{~h}$ glucose levels but lower glucose peak [10]. Furthermore, in a prospective study, we showed associations between glucose patterns and the risk of future diabetes [11]. Now, we propose to use the same statistical method to reveal heterogeneity in plasma glucose patterns based on three time points during the OGTT, and thereafter compare the incidence of not only diabetes but also CVD and all-cause mortality rate between the identified latent classes. We hypothesise that there will be individuals at high risk not captured by classical measures of risk.

\section{Methods}

The analysis was based on data from the Inter99 study, a prospective non-pharmacological intervention study in the Danish population [12]. At baseline, 6784 individuals participated in a clinical examination including a $75 \mathrm{~g}$ OGTT (anhydrous glucose in $250 \mathrm{ml}$ water) with measurement of plasma glucose and serum insulin at three time points (FPG, $30 \mathrm{~min} P G$ and $2 \mathrm{hPG}$ ) after a fast from midnight including drinking and smoking (except for some water to take essential medication in the morning). Altogether, 6171 participants had valid glucose measurements at all three time points. We excluded individuals with known $(n=44)$ or screen-detected diabetes $(n=243)$, leaving 5861 participants for the analysis. Furthermore, 61 individuals with previous CVD were excluded for the analysis of CVD as an outcome variable.

Height, body weight, waist and hip circumference, blood pressure and plasma lipids were measured according to standard procedures. Information on smoking status, diet, physical activity, socioeconomic status and use of medication was obtained by questionnaire. Plasma glucose concentrations were measured using the hexokinase/G6P-DH technique (Boehringer Mannheim, Mannheim, Germany) and serum insulin concentrations by the fluoroimmunoassay technique (AutoDELFIA; Perkin Elmer-Wallac, Turku, Finland).

We calculated the area under the plasma glucose curve (PG-AUC) and the incremental area (PG-iAUC) using the trapezoid rule. As a surrogate marker of insulin sensitivity, we calculated the insulin sensitivity index $\left(\mathrm{ISI}_{0-120}\right)$, a simplified version of the Cederholm index, using the equation by Gutt et al $[13,14]$. We also calculated the insulinogenic index based on fasting and $30 \mathrm{~min}$ glucose and insulin measurements to characterise the insulin response to the glucose challenge [15]. The equations are provided in the electronic supplementary material (ESM).

The Inter99 study was performed in accordance with the Helsinki Declaration with approval by the local ethics committee (KA98155) and informed consent was attained from participants. The Inter99 study has been described in detail elsewhere [12].

Latent class mixed-effects models were fitted to identify distinct patterns of glycaemic response during the OGTT. A piecewise linear specification was used for time terms, allowing coefficients (slopes before and after $30 \mathrm{~min}$ ) to vary between latent classes. The optimal number of classes was selected based on the model's Bayesian Information Criterion, as well as the size of the smallest identified class. Participants were assigned to the class with the highest class membership probability.

For the survival analysis, information on diabetes diagnosis during follow-up (until 31 December 2010) was extracted from the Danish Diabetes Register. Information on CVD (ischaemic heart disease and stroke, until 31 Dec 2011) was obtained from the National Patient Register (non-fatal CVD) and from the Cause of Death Register (fatal CVD). Information on all-cause mortality rate was obtained from the Cause of Death Register on 31 December 2012. Therefore, 11, 12 and 13 years of median follow-up were obtained for the three outcomes, respectively. We had more detailed information on cause of death for those who died before 31 December 2010. Further information on the validation and coverage of the Danish registers has been published previously [16-18]. Cox proportional hazards models with 
adjustment for two sets of variables were used to compare risk of diabetes, CVD and death between latent classes. Model 1 was adjusted for age, sex, smoking status and intervention. Model 2 was additionally adjusted for waist circumference, height, socioeconomic status, diet, physical activity, systolic blood pressure, total cholesterol, antihypertensive medication and statin use. Statistical analyses were conducted in $\mathrm{R}$ version 3.2.0 (R Foundation for Statistical Computing, Vienna, Austria).

\section{Results}

Identification of latent classes Four distinct glucose patterns were identified during the OGTT (Fig. 1a). Average class membership probabilities for the most likely class were high, ranging between 0.8 and 0.9 . Class 3 , representing $13 \%$ of participants, was characterised by the highest $30 \mathrm{minPG}$ but the lowest $2 \mathrm{hPG}$. The other three classes were ranked consistently regarding plasma glucose levels at the three time points.

Cardiometabolic risk factors Characteristics of study participants stratified by latent classes are shown in Table 1. Class 3 had the highest proportion of men and current smokers (both $p<0.001$ vs all other groups) and $>70 \%$ of individuals belonging to this group had normal glucose tolerance. Insulin levels ranked similarly to glucose levels at all three time points. Individuals in class 3 were characterised by the same degree of insulin sensitivity ( $\mathrm{ISI}_{0-120}$ ) as those in class 1 ; however, their first-phase insulin response (insulinogenic in$\operatorname{dex}_{0-30}$ ) was almost reduced to the same extent as those in class 4 . In general, other cardiometabolic risk factors showed a worsening tendency from class 1 to class 4 .

Diabetes Cumulative event probabilities for the different latent classes are shown in Fig. 1b, while absolute numbers and incidence rates with adjusted HRs are provided in Table 2. In the most adjusted model, people in class 3 had a fourfold increased risk of developing diabetes compared with those in class 1 , but only half the risk of those in class 4 (HR 0.48 [95\% CI 0.32 , $0.70], p<0.001)$. Class 3 also had higher diabetes risk than participants in class 2 (HR 1.5 [95\% CI 1.0, 2.2], $p=0.05$ ), who had only one-third of the risk (HR 0.32 [95\% CI 0.23 , $0.45], p<0.001)$ compared with class 4 with the highest incidence rate (15.4 [95\% CI 12.7, 18.6] per 1000 person-years).

CVD We observed smaller differences in risk of developing CVD between latent classes, than in risk of developing diabetes (Fig. 1c and Table 2). Class 3 did not have a higher risk of CVD events than class 1 , but classes 2 and 4 characterised by higher $2 \mathrm{hPG}$ had a 60-70\% higher CVD risk than class 1 in model 1 . However, these effects were attenuated and became non-significant in model 2.
Fig. 1 (a) Patterns of plasma glucose during an OGTT. (b-d) Cumulative incidence proportion (CIP) with $95 \%$ CI of (b) diabetes, (c) CVD and (d) allcause mortality by latent class. Green, class $1(n=1855,32 \%)$ light blue, class $2(n=2534$, $43 \%)$; dark blue, class $3(n=781$, $13 \%)$; red, class $4(n=691,12 \%)$
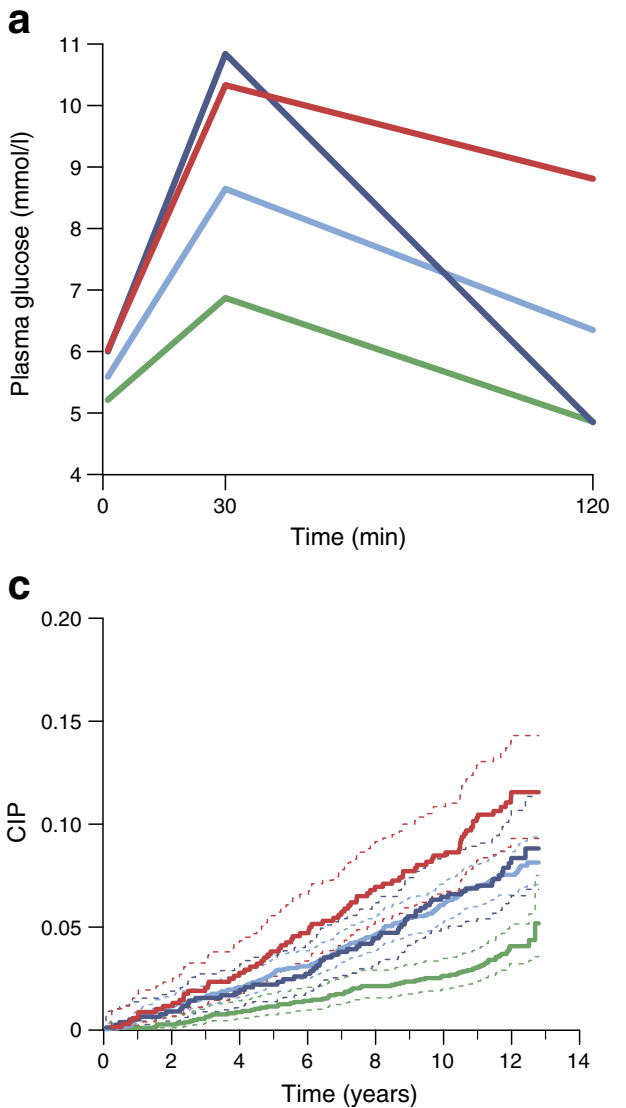

b

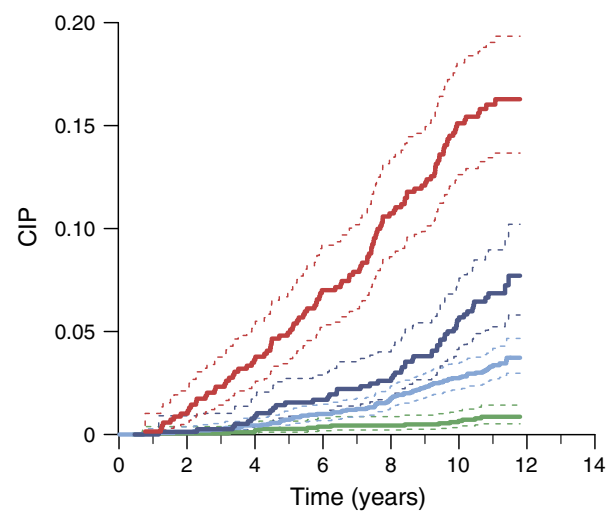

d

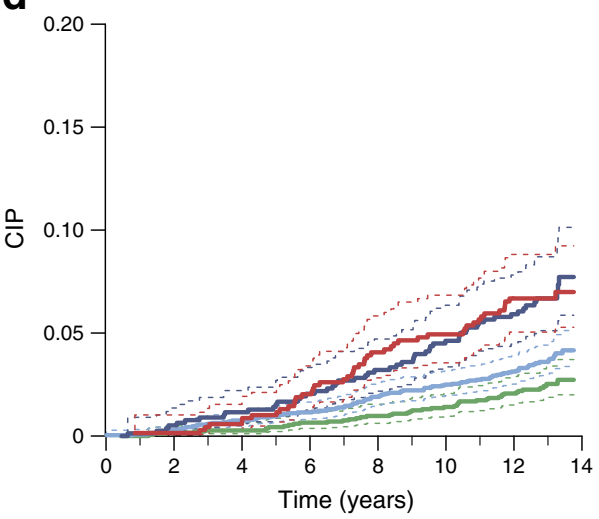


Table 1 Cardiometabolic risk factor characteristics by latent class

\begin{tabular}{|c|c|c|c|c|c|}
\hline & $\begin{array}{l}\text { Overall } \\
n=5861\end{array}$ & $\begin{array}{l}\text { Class } 1 \\
n=1855(32 \%)\end{array}$ & $\begin{array}{l}\text { Class } 2 \\
n=2534(43 \%)\end{array}$ & $\begin{array}{l}\text { Class } 3 \\
n=781(13 \%)\end{array}$ & $\begin{array}{l}\text { Class } 4 \\
n=691(12 \%)\end{array}$ \\
\hline Age (year) & $45(40-50)$ & $45(40-50)$ & $45(40-50)$ & $50(45-55)$ & $50(40-55)$ \\
\hline Sex (\% male) & 50 & 38 & 49 & 76 & 54 \\
\hline BMI $\left(\mathrm{kg} / \mathrm{m}^{2}\right)$ & $25.5(23.1-28.3)$ & $24.2(22.1-26.4)$ & $25.6(23.4-28.6)$ & $26.6(23.8-29.1)$ & $27.7(24.7-31.1)$ \\
\hline Height (cm) & $172(165-179)$ & $171(165-178)$ & $171(165-178)$ & $175(170-182)$ & $171(164-177)$ \\
\hline Waist circumference (cm) & $85(76-94)$ & $80(72-88)$ & $86(77-94)$ & $91(84-99)$ & $92(83-101)$ \\
\hline Hip circumference $(\mathrm{cm})$ & $100(94-106)$ & $98(93-104)$ & $100(95-106)$ & $101(96-107)$ & $102(96-109)$ \\
\hline Total cholesterol (mmol/l) & $5.4(4.8-6.2)$ & $5.1(4.5-5.8)$ & $5.5(4.8-6.3)$ & $5.7(5.0-6.3)$ & $5.8(5.0-6.5)$ \\
\hline LDL-cholesterol (mmol/l) & $3.4(2.8-4.1)$ & $3.2(2.6-3.8)$ & $3.5(2.9-4.2)$ & $3.6(3.0-4.3)$ & $3.6(3.0-4.3)$ \\
\hline HDL-cholesterol (mmol/l) & $1.4(1.1-1.7)$ & $1.4(1.2-1.7)$ & $1.4(1.1-1.7)$ & $1.3(1.1-1.7)$ & $1.3(1.1-1.6)$ \\
\hline Triacylglycerols (mmol/l) & $1.0(0.8-1.5)$ & $0.9(0.7-1.2)$ & $1.1(0.8-1.5)$ & $1.2(0.8-1.7)$ & $1.4(1.0-2.1)$ \\
\hline Systolic blood pressure (mmHg) & $129(120-140)$ & $120(112-130)$ & $130(120-140)$ & $130(120-140)$ & $137(125-150)$ \\
\hline Diastolic blood pressure (mmHg) & $80(75-90)$ & $80(70-85)$ & $80(75-90)$ & $85(79-90)$ & $87(80-95)$ \\
\hline Antihypertensive treatment (\%) & 6 & 3 & 6 & 8 & 11 \\
\hline Smoking $(\%)$ & 36 & 36 & 31 & 50 & 35 \\
\hline Intervention (\%) & 51 & 41 & 47 & 59 & 84 \\
\hline \multicolumn{6}{|l|}{ Glucose tolerance status (\%) } \\
\hline Normal glucose tolerance & 78 & 99 & 86 & 71 & 9 \\
\hline iIFG & 9 & 1 & 8 & 29 & 7 \\
\hline iIGT & 9 & 0 & 5 & 0 & 54 \\
\hline IFG + IGT & 4 & 0 & 1 & 0 & 30 \\
\hline PG-AUC & $14.3(12.7-15.9)$ & $12.0(11.2-12.7)$ & $14.6(13.9-15.5)$ & $15.8(14.7-17.2)$ & $18.2(17.4-19.2)$ \\
\hline PG-iAUC & $3.4(2.2-4.7)$ & $1.6(0.9-2.3)$ & $3.8(3.1-4.6)$ & $4.2(3.3-5.3)$ & $6.6(5.8-7.5)$ \\
\hline Insulin $_{0}(\mathrm{pmol} / \mathrm{l})$ & $33(23-49)$ & $28(20-40)$ & $34(24-49)$ & $35(23-54)$ & $46(30-71)$ \\
\hline Insulin $_{30}(\mathrm{pmol} / \mathrm{l})$ & $243(173-352)$ & $221(160-313)$ & $247(177-351)$ & $273(190-419)$ & $264(172-396)$ \\
\hline Insulin $_{120}(\mathrm{pmol} / \mathrm{l})$ & $151(91-245)$ & $112(72-161)$ & 179 (119-267) & $90(49-161)$ & $353(216-566)$ \\
\hline $\mathrm{ISI}_{0-120}$ & $99(75-128)$ & $125(104-157)$ & $86(73-105)$ & $125(95-176)$ & $52(44-62)$ \\
\hline Insulinogenic index ${ }_{0-30}$ & $0.58(0.36-0.96)$ & $0.91(0.58-1.50)$ & $0.54(0.36-0.83)$ & $0.39(0.25-0.61)$ & $0.38(0.26-0.59)$ \\
\hline
\end{tabular}

Data are presented as median $(\mathrm{Q} 1-\mathrm{Q} 3)$ or percentage

iIFG, isolated impaired fasting glucose; iIGT, isolated impaired glucose tolerance; IFG + IGT, impaired fasting glucose and impaired glucose tolerance

All-cause mortality rate The all-cause mortality rate was higher in classes 3 and 4 than in class 1 (Fig. 1d and Table 2). The differences were attenuated but remained statistically significant after confounder adjustment (model 2). In a subanalysis, we did not find an overall indication of a difference in causes of death by class when considering cancer, CVD and other cause as the three major groups $(p=0.24)$. There was a statistically non-significant indication that CVD was more frequently the main cause of death in class 4 compared with the other classes $(p=0.06)$.

\section{Discussion}

Using a novel latent class trajectory analysis approach, we revealed heterogeneity in plasma glucose response to oral glucose intake beyond the established glucose criteria based on FPG and 2hPG levels. We identified four different patterns and were able to link them to long-term outcomes including incident diabetes, CVD and all-cause mortality rate. While three groups (classes 1,2 and 4) showed a consistently increasing risk profile regarding cardiometabolic risk factors and longterm outcomes, one group (class 3 ) was characterised by a markedly different pattern. The latter had the largest change during the $2 \mathrm{~h}$ OGTT, reaching the highest 30minPG level but then reverting to the lowest $2 \mathrm{hPG}$ level. This group was characterised by having a low first-phase insulin response to the oral glucose challenge but a high level of insulin sensitivity.

Individuals belonging to class $3 \mathrm{had}$ a fourfold higher risk of diabetes than those in class 1 , who had the most favourable pattern. In contrast, class 3 had a lower risk of diabetes than those in class 4 who had a similarly high $30 \mathrm{minPG}$ but higher $2 \mathrm{hPG}$ within the impaired glucose tolerance range. These differences were probably explained by the low first-phase insulin response but relatively high level of insulin sensitivity observed in individuals in class 3 . Intermediate time points, especially 
Table 2 Crude incidence rates (per 1000 person-years) and adjusted HRs $(95 \% \mathrm{CI})$ for diabetes fatal and non-fatal CVD (ischaemic heart disease or stroke) and all-cause mortality by latent class

\begin{tabular}{cclll}
\hline & $n(\%)$ & Incidence rate & HR $(95 \% \mathrm{CI})$ & \\
\cline { 4 - 5 } & & & Model 1 & Model 2 \\
\hline Diabetes & & & \\
Class 1 & $15(0.8 \%)$ & $0.7(0.5,1.2)$ & Ref. & Ref. \\
Class 2 & $83(3.3 \%)$ & $3.0(2.5,3.8)$ & $3.8(2.2,6.6)$ & $2.8(1.6,4.9)$ \\
Class 3 & $52(6.7 \%)$ & $6.3(4.8,8.3)$ & $6.8(3.8,12.2)$ & $4.1(2.2,7.6)$ \\
Class 4 & $108(15.6 \%)$ & $15.4(12.7,18.6)$ & $14.5(8.3,25.4)$ & $8.6(4.7,15.5)$ \\
CVD & & & & \\
Class 1 & $71(3.9 \%)$ & $3.3(2.6,4.2)$ & Ref. & $1.3(1.0,1.8)$ \\
Class 2 & $190(7.6 \%)$ & $6.6(5.7,7.6)$ & $1.6(1.2,2.2)$ & $1.1(0.8,1.7)$ \\
Class 3 & $61(7.9 \%)$ & $6.9(5.4,8.9)$ & $1.3(0.9,1.9)$ & $1.2(0.8,1.8)$ \\
Class 4 & $75(11.0 \%)$ & $9.7(7.8,12.2)$ & $1.7(1.2,2.4)$ & Ref. \\
All-cause mortality & & & & $1.3(0.9,1.9)$ \\
Class 1 & $45(2.4 \%)$ & $1.9(1.4,2.5)$ & Ref. & $1.6(1.0,2.5)$ \\
Class 2 & $95(3.7 \%)$ & $2.9(2.4,3.6)$ & $1.3(0.9,1.9)$ & $1.8(1.1,2.9)$ \\
Class 3 & $54(6.9 \%)$ & $5.5(4.2,7.1)$ & $1.8(1.2,2.7)$ & $1.9(1.2,2.9)$ \\
Class 4 & $47(6.8 \%)$ & $5.4(4.0,7.1)$ & &
\end{tabular}

Model 1: adjusted for age, sex, smoking and intervention

Model 2: adjusted for age, sex, smoking, intervention, waist circumference, height, socioeconomic status, diet, physical activity, systolic blood pressure, total cholesterol, antihypertensive medication and statin use
$1 \mathrm{hPG}$, were also found to be superior markers for future diabetes in other studies $[2,3]$. Our finding is also in line with previous studies, reporting higher diabetes risk among those whose plasma glucose levels did not return to baseline during an OGTT compared with those whose did $[6,11]$. In addition, the patterns identified in the current study were similar to those we identified in an urban Indian population, which supports the use of the latent class trajectory analysis approach [11].

People in class 3 had a CVD risk comparable to the class with the most favourable glucose pattern (class 1). This might be explained by their high level of insulin sensitivity, as a previous study reported an association with low insulin sensitivity. The increased risk of CVD in class 4 was also attenuated in the most adjusted model and became non-significant. This might be due to the classification based on glucose patterns, as two previous large observational studies reported a strong association between low insulin sensitivity and incident CVD when using ISI $_{0-120}$ as a continuous predictor [19, 20]. Although not directly comparable due to the lack of $1 \mathrm{hPG}$ measurements in our study, these results are in agreement with a previous Finnish study finding $2 \mathrm{hPG}$ to be a more important marker of CVD risk than FPG and 1hPG among people with impaired fasting plasma glucose or impaired glucose tolerance [8]. We observed a worsening gradient for most cardiovascular risk factors across classes, but not for smoking. The observed association between the group having a high intermediate peak (class 3 ) and smoking is in line with a previous meta-analysis on the effect of smoking on glycaemic measures and might reflect increased gastric motor activity [21].
In addition to $1 \mathrm{hPG}$ concentration, various curve characteristics have been examined in predicting future diabetes [3, 6]. However, such measures have limitations, as individuals with different glucose trajectories can still have similar values for some or several calculated curve features. Therefore, instead of studying predefined curve characteristics, we considered heterogeneity of change using a data-driven approach. Latent class trajectory analysis allows the investigation of change over time, while taking measurement error into account. Compared with conventional approaches assessing only mean growth curves, the latent class method is suitable to reveal heterogeneous patterns, which may give us a more complete picture of associations. Another strength of our analysis was that we could include not only diabetes but also CVD and all-cause mortality rate as outcomes within the same cohort. This has been a limitation of previous studies, which have focused on diabetes development.

It is important to bear in mind that the aim of this study was not to find the best prediction model or to determine optimal cut-off values for the examined outcomes, but to find associations that might not have been revealed with conventional methods. We observed that different glucose patterns are associated with different long-term outcomes, which cannot be explained by differences in cardiometabolic risk profiles. This suggests that glucose measurements at different time points should be considered simultaneously to get a more detailed picture of a person's risk of future diabetes, CVD and premature death. A limitation of our study was that we were not able to study other intermediate time points during 
the $2 \mathrm{~h}$ OGTT (e.g. 60 or $90 \mathrm{~min}$ ). This would have refined our approach in the sense that continuous glucose curve patterns could be captured, not only piecewise linear. Another aspect that needs further investigation is the difference in mortality rates between classes. Although we had information on the cause of death for a subsample of our cohort, because the numbers in the different categories were low we did not have enough power to examine in detail which causes contributed most to the excess mortality rate in class 3 compared with class 1 . However, the observed difference is in line with previous results showing a higher mortality rate among those with $1 \mathrm{hPG}>8.6 \mathrm{mmol} / 1$ even with a $2 \mathrm{hPG}$ level $<7.8 \mathrm{mmol} / 1$ [9]. Similarly, the low number of individuals with diabetes in class $1(n=15)$ resulted in wide CIs of the HRs when using this class as reference category. Therefore, we also presented some of our results using class 4 , the class with the most events, as reference for this outcome.

In conclusion, elevated $30 \mathrm{~min} P \mathrm{P}$ is associated with a high risk of future diabetes and higher all-cause mortality rates independent of FPG or $2 \mathrm{hPG}$ levels. Latent class analysis seems to be a promising method to reveal otherwise unidentified subgroups that do not fit into either of the categories of impaired fasting glucose or impaired glucose tolerance.

Acknowledgements $\mathrm{AH}$ and DRW are supported by the Danish Diabetes Academy. The Danish Diabetes Academy is funded by the Novo Nordisk Foundation. KF is supported by the Novo Nordisk Foundation.

Data availability The raw data underlying this study are restricted to protect participant privacy as required by data protection acts in Denmark. Data will be made available upon request to researchers who qualify for access to confidential data by contacting The Research Centre for Prevention and Health, the Capital Region of Denmark, Email: fcfs@ regionh.dk.

Funding The Inter99 study was funded by the Danish Research Councils, Health Foundation, Danish Centre for Evaluation and Health Technology Assessment, Copenhagen County, Danish Heart Foundation, Ministry of Health and Prevention, Association of Danish Pharmacies, Augustinus Foundation, Novo Nordisk, Velux Foundation, Becket Foundation, and $\mathrm{Ib}$ Henriksens Foundation. The sponsors were not involved in the design of the study; the collection, analysis and interpretation of data; writing the report; or the decision to submit the report for publication.

Duality of interest The authors declare that there is no duality of interest associated with this manuscript.

Contribution statement $\mathrm{AH}$ was responsible for the data analysis with major contributions from DV. AH wrote the first draft of the manuscript with major contributions from $\mathrm{KF}$. AH, DRW, KF and MB contributed to the original idea for the study. All authors contributed to the interpretation of the data, drafting and critical revision of the manuscript, and approved the final version. $\mathrm{AH}$ is the guarantor of this work.

\section{References}

1. World Health Organization (2006) Definition and diagnosis of diabetes mellitus and intermediate hyperglycemia: report of a WHO/ IDF Consultation. Available from www.who.int/diabetes/ publications/Definition\%20and\%20diagnosis\%20of\%20diabetes new.pdf. Accessed 21 Sep 2017

2. Abdul-Ghani MA, Williams K, DeFronzo RA, Stern M (2007) What is the best predictor of future type 2 diabetes? Diabetes Care 30:1544-1548

3. Alyass A, Almgren P, Akerlund M et al (2015) Modelling of OGTT curve identifies $1 \mathrm{~h}$ plasma glucose level as a strong predictor of incident type 2 diabetes: results from two prospective cohorts. Diabetologia 58:87-97

4. Jagannathan R, Sevick MA, Li H et al (2016) Elevated 1-hour plasma glucose levels are associated with dysglycemia, impaired beta-cell function, and insulin sensitivity: a pilot study from a real world health care setting. Endocrine 52:172-175

5. Bergman M, Chetrit A, Roth J, Dankner R (2015) Dysglycemia and long-term mortality: observations from the Israel study of glucose intolerance, obesity and hypertension. Diabetes Metab Res Rev 31: 368-375

6. Abdul-Ghani MA, Lyssenko V, Tuomi T, Defronzo RA, Groop L (2010) The shape of plasma glucose concentration curve during OGTT predicts future risk of type 2 diabetes. Diabetes Metab Res Rev 26:280-286

7. Kanauchi M, Kimura K, Kanauchi K, Saito Y (2005) Beta-cell function and insulin sensitivity contribute to the shape of plasma glucose curve during an oral glucose tolerance test in non-diabetic individuals. Int J Clin Pract 59:427-432

8. Lind M, Tuomilehto J, Uusitupa M et al (2014) The association between $\mathrm{HbA}_{1 \mathrm{c}}$, fasting glucose, 1-hour glucose and 2-hour glucose during an oral glucose tolerance test and cardiovascular disease in individuals with elevated risk for diabetes. PLoS One 9:e109506

9. Bergman M, Chetrit A, Roth J, Dankner R (2016) One-hour postload plasma glucose level during the OGTT predicts mortality: observations from the Israel Study of Glucose Intolerance, Obesity and Hypertension. Diabet Med 33:1060-1066

10. Hulman A, Simmons RK, Vistisen D et al (2017) Heterogeneity in glucose response curves during an oral glucose tolerance test and associated cardiometabolic risk. Endocrine 55:427-434

11. Hulman A, Gujral UP, Narayan KMV et al (2017) Glucose patterns during the OGTT and risk of future diabetes in an urban Indian population: the CARRS study. Diabetes Res Clin Pract 126:192197

12. Jorgensen T, Borch-Johnsen K, Thomsen TF, Ibsen H, Glumer C, Pisinger C (2003) A randomized non-pharmacological intervention study for prevention of ischaemic heart disease: baseline results Inter99. Eur J Cardiovasc Prev Rehabil 10:377-386

13. Cederholm J, Wibell L (1990) Insulin release and peripheral sensitivity at the oral glucose tolerance test. Diabetes Res Clin Pract 10: 167-175

14. Gutt M, Davis CL, Spitzer SB et al Validation of the insulin sensitivity index ${ }_{0,120}$ : comparison with other measures. Diabetes Res Clin Pract 47:177-184

15. Seltzer HS, Allen EW, Herron AL Jr, Brennan MT (1967) Insulin secretion in response to glycemic stimulus: relation of delayed initial release to carbohydrate intolerance in mild diabetes mellitus. J Clin Invest 46:323-335

16. Carstensen B, Kristensen JK, Marcussen MM, Borch-Johnsen K (2011) The National Diabetes Register. Scand J Public Health 39: 58-61

17. Helweg-Larsen K (2011) The Danish Register of causes of death. Scand J Public Health 39:26-29 
18. Lynge E, Sandegaard JL, Rebolj M (2011) The Danish National Patient Register. Scand J Public Health 39:30-33

19. Rutter MK, Meigs JB, Sullivan LM, D'Agostino RB Sr, Wilson PW (2005) Insulin resistance, the metabolic syndrome, and incident cardiovascular events in the Framingham Offspring Study. Diabetes $54: 3252-3257$
20. Zethelius B, Cederholm J (2015) Comparison between indexes of insulin resistance for risk prediction of cardiovascular diseases or development of diabetes. Diabetes Res Clin Pract 110:183-192

21. Soulimane $\mathrm{S}$, Simon D, Herman WH et al (2014) $\mathrm{HbA}_{1 \mathrm{c}}$, fasting and $2 \mathrm{~h}$ plasma glucose in current, ex- and never-smokers: a metaanalysis. Diabetologia 57:30-39 